\title{
AN ANAEROBIC BACILLUS ISOLATED FROM A CASE OF CHRONIC BRONCHITIS.*
}

\author{
R UTH T UNNICLIF F. \\ (From the Memorial Institute for Infectious Diseases, Chicago.)
}

In making bacteriological examinations of the sputum from a case of chronic bronchitis, suspected of being tuberculous, an anaerobic bacillus was found to be the predominating organism. So far as I have been able to determine, this bacillus has not been previously described.

The patient is a man 62 years old. Until four years of age he was perfectly healthy. At that time he had a severe attack of pneumonia, followed by repeated attacks of lobar and bronchopneumonia.

About 40 years ago the patient began to suffer probably from tuberculosis, having repeated attacks of chills, fever, and sweating for eight years, getting better for a year and then worse again. At this time he had a very severe attack of lobar pneumonia, and a pronounced pleurisy with effusion. Since then he has suffered from similar attacks of pleurisy with more or less bronchitis; I9 years ago he had a very severe attack of pleurisy with effusions lasting several weeks; these repeated attacks of bronchitis and pleurisy, which always disappeared after a few weeks, persisted until four years ago, since which time only bronchitis has been troublesome. Six months ago the patient was ill with an attack of bronchitis, much worse than those previous, and continued with a profuse expectoration until the vaccine treatment now to be described was employed.

The bacillus in the sputum is about two microns long by onefourth micron in width. It was found in enormous numbers generally in clumps. Some bacilli were seen inside of leukocytes. They were found as a rule in greyish masses in the sputum. Altho resembling tubercle bacilli a good deal, they were not acidfast nor did they retain Gram's stain. They stained uniformly

*Received for publication June 9 , 19.3. 
and deeply with carbol-gentian-violet, faintly with methylene blue, having a beaded appearance, as they did with carbol-fuchsin. With Giemsa they stained blue.

The bacillus is a strict anaerobe, growing slowly, especially during the first generations. The first time it was isolated, growth did not begin until the fifteenth day after inoculation. It was isolated on goat blood agar, the agar being slightly alkalin to phenolphthalein. It grew in the fluid of condensation as a thick mucoid mass. On the surface of the agar profuse thick yellowish colonies appeared, containing the bacillus mixed with other bacteria. In subcultures the bacillus was isolated in pure culture, the colonies now appearing after five days' incubation. The colonies are round, convex and white, looking like pearls. As they grow older they become yellower in color. They are hard and can be removed only by also removing the agar. They may be pierced by a stiff needle. They grow in the fluid of condensation as a greyish-white mucoid mass, or the organisms may grow as distinct balls, held in a mucoid mass. In subcultures the colonies may appear in 48 hours as pinpoint, hard, dull colonies, increasing gradually in size and becoming white as they enlarge. The bacillus was isolated in pure culture a second time, the colonies appearing then on the eighth day, as separate pearl-white hard colonies. The bacillus after five months' isolation is still a strict anaerobe and has not become acidfast.

The bacillus grows in 2 per cent dextrose broth in a creamy irregular mucoid mass, the fluid remaining perfectly clear. .At first it would not grow on the surface of ascites agar and Loeffler's blood serum, only growing in the fluid of condensation as in the dextrose broth. The colonies on ascites and glycerin agar appear like those on blood agar, but those on Loeffler's blood serum are a little softer. On potato the colonies are a little.more irregular in shape, especially as they get older. The edge of the colonies as a rule is smooth. The colonies adhere to the media, but do not appear to grow far into it. The bacillus does not grow on plain agar, plain broth, milk, dextrose or mannite agar.

The bacillus is not motile and does not possess a capsule. When grown on potato and Loeffler's blood serum, it appears larger than when grown on blood agar and is more curved and shows 
many bizarre forms. When grown on alkalin goat-blood agar or ordinary blood agar, it appears as a short, fairly thick bacillus of the same size and staining properties as the bacillus found in the smears from the sputum. Filaments are sometimes seen in the cultures. They are also seen in the sputum.

If inoculated in large numbers the bacillus appears to be toxic to guinea-pigs, but not pathogenic. The sputum injected into a guinea-pig did not produce any lesions. A guinea-pig was injected in the right lung with the growth in the fluid of condensation from

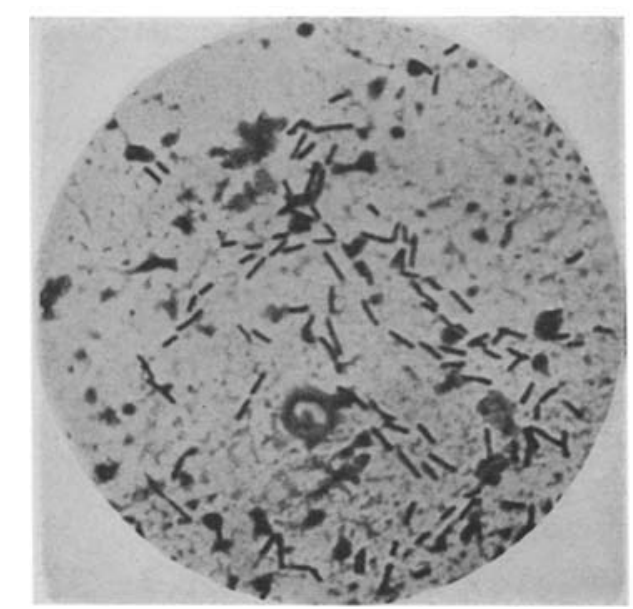

Fig. I.-Smear from sputum. $\times$ r200. Carbolgentian-violet.

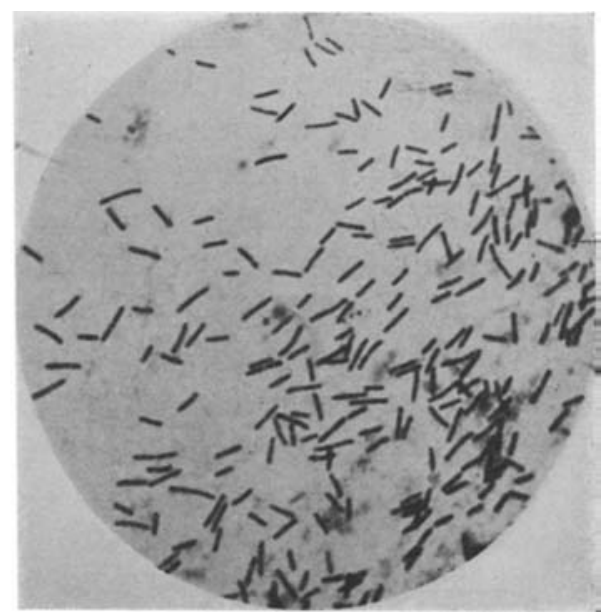

Fig. 2-Pure culture, three days old. $\times 1200$. Carbol-gentian-violet.

two tubes of Loeffler's blood serum grown 48 hours. Eighteen days later the same pig was injected in the left lung with the growth in the fluid of condensation of three tubes of Loeffler's blood serum. Immediately after this injection the guinea-pig had great difficulty in breathing, lying on the right side and twitching considerably. It had some trouble in breathing and appeared sick for four days. Ten days after the second injection the guinea-pig was killed. Areas of atelectasis were found in both sides, and a small area of congestion was seen in the middle right lobe. This proved to be chronic bronchopneumonia.

Another guinea-pig (small) was injected in the lung with the 
growth in the fluid of condensation of two tubes of Loeffler's blood serum. In seven days it died in convulsions. No pathological lesions could be found.

Repeated intraperitoneal injections in a guinea-pig have so far produced no effect.

On account of the chronic condition of the bronchitis, the presence of cavities in the lung as determined by physical examination, and the profuse expectoration, it was thought a vaccine of this bacillus might prove of value. The colonies were dug out of the blood agar, suspended in normal salt solution, and ground in a sterile mortar. This suspension was centrifuged to remove the pieces of agar and then heated for one hour at $60^{\circ} \mathrm{C}$. Its sterility was determined by cultures and animal inoculation. The patient's opsonic index was determined before and after the inoculations. The bacilli for these experiments were also ground in a mortar to get suitable suspensions. The bacilli are practically not taken up at all in the presence of normal serum, as a rule about four cells out of 50 taking part in phagocytosis. The patient had an index slightly higher than normal before the injections began. After each injection the changes in the serum were most marked, as seen both in the number of bacteria ingested by each leukocyte, and by the number of cells taking part in phagocytosis. The indices after the injections ranged from 18 to 38 . After the first two injections there was a negative phase lasting four days after the first, and one day after the second. No observations were made after the third injection. There was no negative phase as indicated by the opsonic index, after the fourth injection.

Five hundred thousand killed bacilli were injected intramuscularly each time, about one month elapsing between each injection. The symptoms were marked after each injection and hence a larger dose was not considered advisable. After each injection there was headache, malaise, sometimes chilliness, the appearance of rales (sibilant and mucous) with increased cough and expectoration. After the first and third injections a little blood appeared in the sputum. About four days after the first injection the patient began to feel much better, sleeping better than for weeks, and having much less bronchitis and therefore decreased expectoration. 
After the second injection when the index rose to 38 , the patient was better than he had been for six months. The amount of sputum was greatly decreased, being less than one-tenth of the amount it had been before the injections. The sputum was more mucoid and less purulent in character. After the third injection the patient had about the same reaction as after the first. When this had passed off the bronchitis was much improved and he felt better again and gained in weight.

The bacilli are still present in the sputum, altho the amount of sputum is greatly reduced.

Whether the vaccine will be able to overcome the infection in the cavities is, of course, doubtful. There can be little doubt, however, that the injections have greatly improved the condition of the patient, the bronchitis being much diminished.

The large number of bacilli present in the sputum, the decided local reaction in the lungs after each injection, and the improvement in the bronchitis after the vaccine treatment would indicate that this organism may have some causal relation with the bronchial infection. 